\section{Septicaemic low birthweight neonates treated with human antibodies to endotoxin}

Sir,

We were encouraged by the study of Adhikari et al ${ }^{1}$ which showed a significant reduction in recovery time from proved sepsis in babies less than $2.5 \mathrm{~kg}$ birthweight treated with an anti-lipopolysaccharide gammaglobulin. As the authors comment, in the acutely ill septicaemic baby, absorption from an intramuscular injection may have been too slow to produce any difference in mortality in the treated group.

Babies of gestational age 32 weeks or less have been shown to be the group most susceptible to immunoglobulin deficiency. ${ }^{2}$ A greater benefit from treatment might have been apparent in Adhikari's study by restricting analysis to such a group. We suggest that all such babies might benefit from weekly intramuscular gammaglobulin as prophylaxis against the onset of severe infection. Such a project is at present under study in our unit.

We question the giving of placebo intramuscular injections to neonates. The pain factor is too often ignored in the management of preterm and low birthweight babies. We feel that further studies on the lines of the paper by Adhikari should be reserved until intravenous prepara- tions are available and their administration confined to those babies ill enough to require an intravenous infusion.

References

' Adhikari M, Coovadia HM, Gaffin SL, Brock-Utne JG, Marivate M, Pudifin DJ. Septicaemic low birthweight neonates treated with human antibodies to endotoxin. Arch Dis Child 1985;60:382-4.

2 Conway SP, Dear PRF, Smith I. Immunoglobulin profile of the preterm baby. Arch Dis Child 1985;60:208-12.

S P Conway, D R N Gillies, and P J Congdon The General Infirmary, Leeds LS2 9NS

Dr Adhikari and Professor Coovadia comment:

We agree with the opinions expressed by Drs Conway, Gillies, and Congdon and thank them for their interest in our paper. We are about to commence a trial of intravenous polyvalent gammaglobulin in 'at risk' low birthweight babies. The specific intravenous anti-lipopolysaccharide gammaglobulin is awaiting the Medicine Council's approval and when available a controlled trial will be conducted.

Our numbers of babies less than 32 weeks' gestation are too small for statistical analyses but there is a trend to a longer period of survival and a shorter time to recovery in the treated groups. 